\title{
Anabases
}

ANABASES Traditions et réceptions de l'Antiquité

$24 \mid 2016$

Varia

\section{Le langage des époux grecs : lecture de quelques inscriptions}

Michela Costanzi

\section{(2) OpenEdition}

Journals

Édition électronique

URL : http://journals.openedition.org/anabases/5931

DOI : 10.4000/anabases.5931

ISSN : 2256-9421

Éditeur

E.R.A.S.M.E.

Édition imprimée

Date de publication : 10 novembre 2016

Pagination : 125-138

ISSN : 1774-4296

\section{Référence électronique}

Michela Costanzi, «Le langage des époux grecs : lecture de quelques inscriptions », Anabases [En ligne], 24 | 2016, mis en ligne le 01 octobre 2019, consulté le 23 mars 2020. URL : http://

journals.openedition.org/anabases/5931; DOI : https://doi.org/10.4000/anabases.5931

(c) Anabases 


\section{Le langage du couple grec : lecture de quelques inscriptions ${ }^{1}$}

\section{Michela Costanzi}

\footnotetext{
$\mathrm{L}$
} e langage du couple grec a laissé des échos dans les sources littéraires des Ve et $\mathrm{IV}^{\mathrm{e}} \mathrm{s}$. av. J.-C., surtout dans les drames, les tragédies et les comédies, et dans les plaidoyers, amplement étudiés ${ }^{2}$, sur lesquels je ne vais donc pas revenir. Mais de ce langage, il reste aussi des traces dans les inscriptions, sur lesquelles j'ai choisi de me concentrer pour différentes raisons. La première est que dans la bibliographie moderne sur la question, une étude systématique sur ce sujet n'a jamais été réalisée. Certes, une abondante littérature existe sur le mariage grec ${ }^{3}$, ainsi que sur le rôle de la femme ${ }^{4}$; mais aucune de ces études n'aborde la question du langage du couple. J'ai pensé par ailleurs que c'était peut-être là que j'allais pouvoir trouver une forme d'expression plus directe, et pouvoir reconnaître une certaine spontanéité, en dépit des codes sociaux et stylistiques dont l'écriture pétrifiée est souvent empreinte.

Les sources les plus importantes utilisées dans ce travail, accompli sans aucune réelle compétence dans les gender studies, mais avec le seul intérêt que j’ai

1 Je remercie Hugues Berthelot d'avoir accepté de relire cet article.

2 A.-M. Verilhac, Cl. Vial, "Les sources », in A.-M. Verilhac, Cl. Vial, Le mariage grec du VIe siècle av. J.-C. à l'époque d'Auguste, BCH Suppl. 32 (1998), p. 15-39.

3 Verilhac, Vial, Le mariage grec du VIe siècle av. J.-C. à l'époque d'Auguste, op. cit.

4 Pour en citer quelques-uns : Cl. Mossé, La femme dans la Grèce antique, Bruxelles, 1990 ; A. Bielman, Femmes en public dans le monde hellénistique : $I^{e}-I^{e r}$ s. av. J.-C., Paris, 2002 ; N. Bernard, Femmes et société dans la Grèce classique, Paris, 2003 ; P. Brulé, Les femmes grecques à l'époque classique, Paris, 2010 ; S. Boenringer, V. SebillotteCuchet, Hommes et femmes dans l'Antiquité, Paris, 2011 ; Femmes et Actes de mémoire. La temporalité dans les échanges, Pallas 99 (2015). 
pour l'épigraphie, sont donc les recueils d'inscriptions funéraires ${ }^{5}$ auxquels s'en ajoutent d'autres d'un autre genre' .

A partir de tels corpora, une étude sur l'image des femmes dans les épigrammes funéraires grecques avait été publiée il y a quelques années ${ }^{7}$, mais se limitait aux inscriptions dédiées à celles-ci. Or il existe aussi des inscriptions dédiées par les femmes à leurs maris, dont je souhaite présenter quelques exemples, qui ne relèvent pas uniquement du genre de l'épitaphe. Un article intéressant pour les idées qu'il offre est celui de G. Raepsaet ${ }^{8}$, mais cet auteur se borne aussi à faire référence aux inscriptions funéraires, alors qu'il existe des inscriptions d'une autre fonction, dont je vais donner un échantillon.

Loin d'être une étude exhaustive sur le langage du couple, cet article a donc la modeste intention de présenter un choix d'inscriptions de types différents : surtout des épigrammes funéraires, bien sûr; je présenterai également des inscriptions privées et des inscriptions publiques, d'autant plus que ces textes semblent parler de couples bien vivants.

Ces textes datent de l'époque classique à l'époque impériale, et proviennent de différentes parties du monde grec : de la Grèce propre (l'Attique, la Béotie, et Delphes), de Rhodes et de la Libye, et de Rome.

Si à l'époque archaïque les couples ne semblent pas exprimer leurs sentiments au travers des inscriptions, l'époque classique nous livre ce que nous appellerons des « textes de couple ». Il s'agit généralement d'inscriptions funéraires, gravées sur des stèles et qui, de ce fait, sont arrivées plus facilement jusqu'à nous. Cependant, un vase du Louvre offre un exemple qui montre que les couples grecs pouvaient s'adresser des phrases inscrites sur des objets de la vie quotidienne,

5 A. Conze, Die attischen Grabreliefs, Berlin-Leipzig, 1880-1922 (abr. Conze) ; W. Peek, Griechische Vers-Inscriften, Berlin, 1955 (abr. Pевк GV); Griechische Grabgedichte, Berlin, 1960 (abr. Peek GG); Ch. W. Clairmont, Gravestone and Epigram. Greek Memorials from the Archaic and Classical Period, Mainz on Rhine, 1970 (abr. Clairmont) ; G. Daux, “Stèles funéraires et épigrammes », BCH 96, 1 (1972), p. 503-566.

6 En particulier, j'ai utilisé $I G, S E G, B E$; W. Froenner, Les inscriptions grecques interprétées, Paris, 1880 (abr. Froenner) ; Fr. Preisigke et alii, Sammelbuch griechischer Urkunden aus Ägypten, 1-3, Berlin-Leipzig, 1913-1927 (abr. SB), M. Guarducci, L'epigrafia greca dalle origini al tardo impero, Rome, 1987 (abr. GuARducci 1987) et Fr. LefÈvre, Corpus des Inscription de Delphes. IV. Les documents amphictioniques, Athènes-Paris, 2002 (abr. CID IV).

7 A.-M. Verilhac, "L'image de la femme dans les épigrammes funéraires », in La femme dans le monde méditerranéen I. Antiquité, Lyon (Maison de l'Orient et de la Méditerranée Jean Pouilloux), 1985, p. 85-112.

8 G. Raepsaet, "Sentiments conjugaux à Athènes aux $\mathrm{V}^{\mathrm{e}}$ et $\mathrm{IV}^{\mathrm{e}} \mathrm{s}$. avant notre ère ", Antiquité Classique, 50, 1-2 (1981), p. 677-684. 
dont on peut penser que leur fragilité a empêché qu'ils arrivent plus nombreux jusqu'à nous.

\section{Inscription sur vase}

Inscription de Mogéa à Eukharis (milieu du $\mathrm{V}^{\mathrm{e}} \mathrm{s}$.)

IG VII, 3467

GuARDUCCI 1987, p. 362-363

L'inscription est incisée en graffiti sur un canthare en argile, à vernis noir, retrouvé à Thespies, en Béotie, et datant du milieu du V V s., conservé au Musée du Louvre.

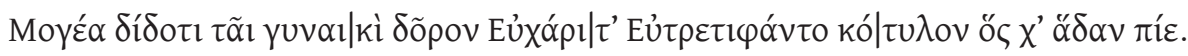

“Mogéa donne comme cadeau à sa femme Eukharis, fille d'Eutrétiphantos ce cotyle afin qu'elle boive à satiété ${ }^{\prime}$ ".

Cette inscription a probablement été gravée directement par le mari, sur un objet de la maison, que le couple pouvait utiliser tous les jours, ou exposer pour faire rire les invités, et montre en effet le sens de l'humour du mari, qui peutêtre trouvait sa femme un peu trop sage. Elle montre un échange plutôt affable entre mari et femme, dans le cours de la vie quotidienne : les époux se moquent, s'amusent.

Mais si cette inscription révèle un aspect de la vie quotidienne que mènent les deux époux, les sentiments du couple s'expriment surtout dans les inscriptions funéraires qui sont arrivées en nombre jusqu'à nous, grâce à la résistance de leur support, et parce que le moment de la mort était apparemment très favorable aux manifestations affectives adressées au conjoint.

Comme Alain Pasquier l'a rappelé dans le présent recueil, les stèles funéraires étaient souvent fabriquées à l'avance. Elles étaient ensuite achetées au moment où on en avait besoin, sans que la représentation ait une relation nécessaire avec la personne défunte. Donc, il ne s'impose pas de chercher des rapports entre la représentation figurée et le texte gravé. Les inscriptions, en revanche, sont réalisées pour la personne morte, et expriment des sentiments et des sensations liés à cette personne. En présentant un petit choix de ces inscriptions funéraires, choix dicté surtout par l'exigence de proposer un échantillon de mots exprimant les sentiments du couple, je voudrais simplement faire remarquer que si, aux $\mathrm{V}^{\mathrm{e}}$ et $\mathrm{IV}^{\mathrm{e}} \mathrm{s}$., ce sont surtout des maris qui rendent hommage à leur femme disparue (ce qui peut paraitre normal, car on imagine pour les femmes quelque difficulté à disposer de l'argent nécessaire à l'achat d'une stèle et au paiement des services

9 Les traductions, sauf indication différente, sont les miennes. 
d'un lapicide), il convient cependant de ne pas oublier qu'à la même période on rencontre aussi des inscriptions où ce sont les femmes qui honorent le souvenir de leur mari.

\section{Inscriptions dédiées à des femmes par leurs maris}

Inscription de Ménékratès à sa femme Chairestratè (milieu du $\mathbf{I V}^{\mathrm{e}} \mathbf{s}$.)

Conze 95, pl. 37

$I G \mathrm{II} / \mathrm{III}^{2} 6288$

PEEK GV 421

Clairmont 26, pl. 13

La stèle est fragmentaire : la partie haute et la base sont perdues. L'inscription se compose de 4 hexamètres sur 5 lignes.

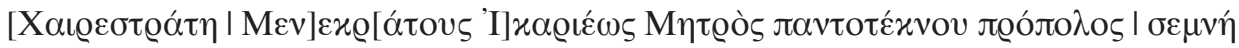

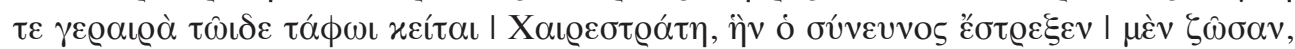

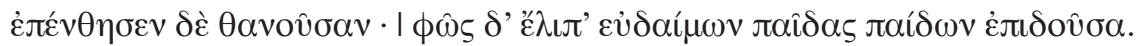

“ Khairéstratè (femme? fille ?) de Ménékratès du dème d'Ikaria, servante de la Mère de tous les Dieux et vénérable vieillarde, c'est dans cette tombe qu'elle gît, Khairéstratè, que son mari concubin chérissait quand elle était en vie et qu'il pleure à présent qu'elle est morte ; la lumière du jour, elle l'a quittée, bienheureuse, elle qui a contemplé les enfants de ses enfants ».

L'épiclèse utilisée pour Khairéstratè est normalement attribuée aux prêtresses du culte de Cybèle. Il s'agit ici d'une Athénienne prêtresse de ce culte, qui est mère et grand-mère et donc vieille et vénérable. Il faut remarquer qu'en général les épigrammes funéraires s'adressent à des jeunes femmes qui n'ont même pas pu avoir des enfants, ou alors à des femmes qui ont pu donner vie à une grande famille. Le mari ici est “ celui qui partage le lit », qu’on dit aussi alochos, ou akoitès, et non pas le posis, mot à consonance poétique. Le verbe pour indiquer le sentiment qui unissait cette femme à son mari quand elle était en vie est stergein (chérir) : il est utilisé pour parler des sentiments conjugaux chez Hérodote, Eschyle et Euripide, mais surtout il indique le sentiment que manifestent les parents à l'égard de leurs enfants et réciproquement ; il conviendrait très bien au statut de mère et grandmère. L'autre verbe très fréquent est philein, tandis qu'eran, (aimer avec passion) n'est jamais présent sur ces stèles ; les sentiments conformes à la bienséance, sont modérés et sans excès. Le mari ne peut que pleurer sa femme maintenant qu'elle est morte (penthein, porter le deuil). 
Inscription d'Antiphilos à sa femme Dionysia (vers 350 a.C.)

Conze 858, pl. 166

IG II/III 11162

Peek $G V 1810, G G 92$

Clairmont 20, pl. 10

Deux fragments composent une stèle, retrouvés au Pirée et actuellement au Musée Archéologique d'Athènes.

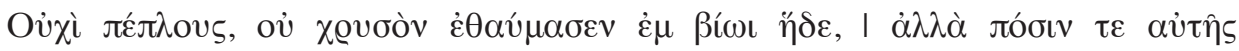

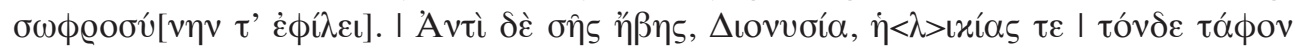

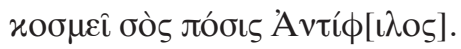

“ Ni les robes, ni l'or, elle n'admira au cours de sa vie, mais son mari et la modération, elle les aimait. A la place de ta jeunesse, Dionysia, de ta maturité, c'est ce tombeau qu'orne ton époux, Antiphilos ».

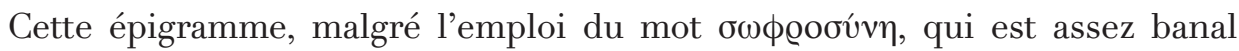
parmi les qualités attribuées aux femmes mortes, semble assez personnelle ; la phrase qu'Antiphilos fait graver pour sa femme est originale : elle n'aimait pas les choses frivoles, mais elle aimait la modération, et surtout son mari. Nous pouvons remarquer qu'ici l'inscription utilise deux mots qui indiquent deux phases de la vie d'une personne, í $\beta \eta$ et $\dot{\eta}<\lambda>\iota x i ́ \alpha$ : l'hébé indique l'adolescence, qui se conclut à 16 ans à Athènes, à 18 ans à Sparte, à 14 ans ailleurs ; quant à l'hélikia, c'est l'âge mûr, celui auquel la femme a normalement des enfants. On doit donc en déduire que cette fille est morte très jeune, avant même le terme de son hébé, peut-être avec un soupçon d'exagération poétique, et qu'elle n'a pas pu atteindre l'âge d'avoir des enfants. Le mari est le posis, et l'amour est en réalité indiqué avec le verbe philein (aimer avec affection).

\section{Inscription de Damoclès à sa femme Kalliarista (premier quart du $\mathbf{I V}^{\mathrm{e}} \mathbf{s}$. )} Peek GV 893

Clara Rhodos IX (1938), 81 ff., pl. 7, fig. 49-50

REG 59-60 (1946-1947), 338, n. 158

Clairmont 32, pl. 16

La stèle, trouvée à Rhodes, où elle est conservée, est presque intacte. Elle est en marbre de Paros et de style attique ; mais les stèles attiques n’ont presque jamais été retrouvées en dehors de l'Attique. Il est possible qu'on ait fait ériger à Rhodes ce monument par un artiste athénien ou formé à Athènes. L'inscription court sur deux lignes, et comporte 3 hexamètres suivis de 2 pentamètres.

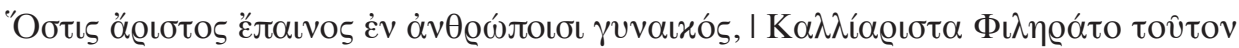

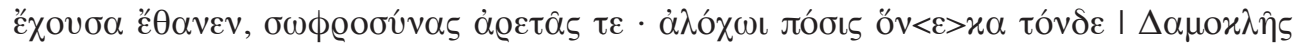

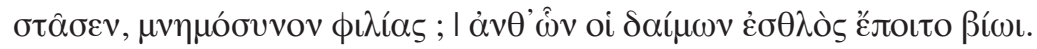


“ Le meilleur éloge du monde pour une femme, Kalliarista fille de Philératos l’a eu à sa mort, pour sa modération et sa vertu ; pour son épouse, pour ces raisons, son époux Damoklès l'a fait dresser en souvenir de son amour ; en échange puisse son esprit fidèle (de la défunte) le suivre dans sa vie ».

La dernière phrase montre un certain intérêt du mari, qui demande en échange de la stèle un destin favorable, auquel l'âme de la morte, ainsi satisfaite, peut contribuer. L'échange entre les époux peut ainsi continuer par-delà la tombe.

Inscription de Mélitè, femme d'Onèsimos (ca 350 a.C.)

Conze 162, pl. 51

$I G \mathrm{II} / \mathrm{III}^{2} 12067$

Реeк GV 1387, GG 101

Clairmont 39, pl. 19

Inscription retrouvée au Pirée.

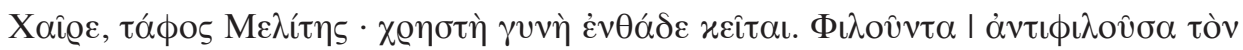

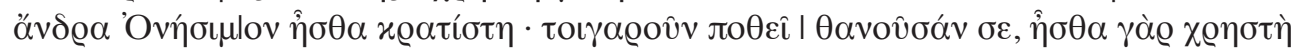

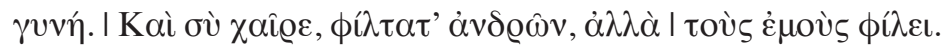

"Salut, tombeau de Mélitè ! C'est une femme de qualité qui gît ici. Aimant en retour un mari qui t'aimait, Onèsimos, tu étais la meilleure (des femmes) ; voilà pourquoi il te regrette, à présent que tu es morte, car tu étais une femme de qualité. Et toi, salut, le plus aimé des hommes, et aime les miens ! ».

L'inscription compte 6 lignes et demi ; entre chaque vers l'espace d'une lettre est laissé comme pour marquer le début du vers suivant. Onèsimos dédie la stèle à sa femme Mélitè. Les trois premiers vers sont des mots d'Onèsimos, mais au vers 4 Mélitè elle-même répond à son mari, en le rassurant sur son amour et en lui rappelant d'aimer ceux qu'elle aime.

Cette épigramme est alors un petit dialogue, et comme dans le cas précédent, la mort ne met pas un terme à la relation. La formule philein antiphilein est utilisée par deux auteurs, Platon (Phèdre 255d) et Aristote (Ethique à Nicomaque 1155b28, 1157b30, 1159a30).

Inscription de Moskhos à sa femme Myrtis (milieu du IV ${ }^{\mathrm{e}}$ s. environ) $I G \mathrm{II} / \mathrm{III} \mathrm{I}^{2}$ 12210a

PeEk GV 343

Clairmont 40, pl. 20

L'inscription, de provenance inconnue, est conservée à l'Institut Archéologique de Leipzig. Elle est en marbre pentélique. 


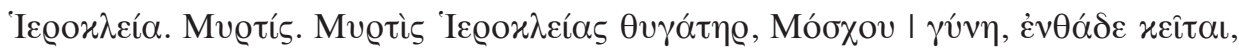

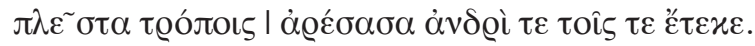

“ Hiérokléia. Myrtis. Myrtis, fille de Hiérokléia, femme de Moskhos, ici gît, qui par son caractère plaisait énormément tant à son mari qu'à ceux qu'elle a enfantés ».

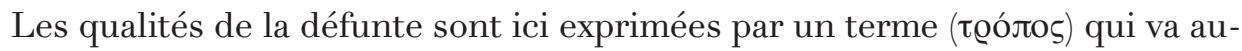
delà des notions traditionnelles de modestie et de retenue : c'est l'ensemble de sa personnalité et de son comportement qui est regretté.

\section{Un exemple d'inscription dédiée par une femme à son mari}

Inscription d'Erxis à son mari Démétrios (vers le milieu du IV ${ }^{\mathrm{e}} \mathbf{s}$.) Conze 191, pl. 54

$I G \mathrm{II} / \mathrm{III}^{2} 11103$

Peek GV 1963

Clairmont 42, pl. 21

Cette inscription, de provenance inconnue, est conservée au Musée National d'Athènes.

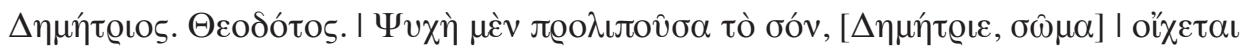

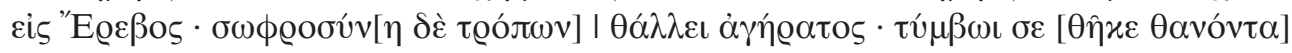

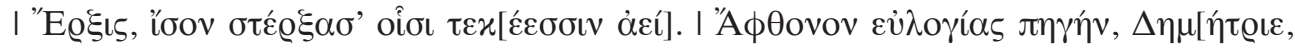

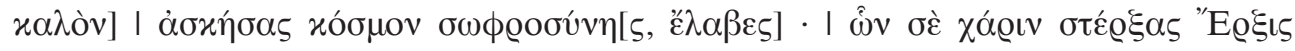

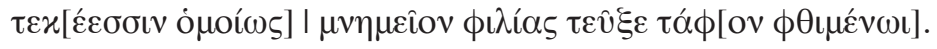

“ Démétrios. Théodotos. Ton âme, quittant ton corps, Démétrios, part pour l'Erèbe ; la modération de ton caractère fleurit, elle qui est sans âge ; c'est au tombeau que t'a placé, à ta mort, Erxis, qui t'a toujours chéri autant que ses enfants. C'est une source abondante d'éloge que tu as gagnée, Démétrios, pour avoir adopté une bonne attitude de modération ; t'ayant pour ces raisons chéri comme ses enfants, Erxis a fait faire, souvenir de votre amour, un tombeau pour toi qui es mort ».

C'est ici une épigramme dédiée par une femme à son mari, dont on apprécie la sophrosuné, la modération, qualité qui n'est donc pas exclusivement féminine (cf. supra les inscriptions de Dionysia et Kalliarista). Le verbe qui exprime l'amour est encore stergein. 


\section{Quelques inscriptions de Libye}

Pour le IV ${ }^{\mathrm{e}} \mathrm{s}$., une inscription de Barca, en Libye, montre le même langage, les mêmes mots, mais aussi la même intention, toujours renouvelée, d'exprimer la douleur de la perte d'une personne aimée ${ }^{10}$.

Inscription d'Arata (Barca, milieu du IV ${ }^{\mathrm{e}} \mathrm{s}$.)

SEG 9, 362

PeEK GV 1912, GG 444

SEG 37, 1714

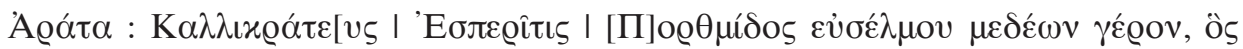

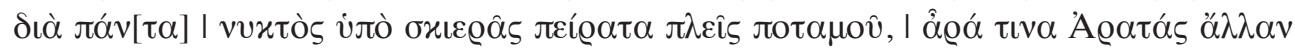

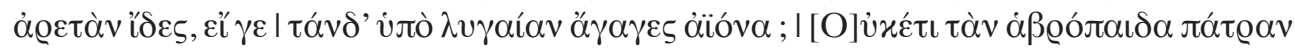

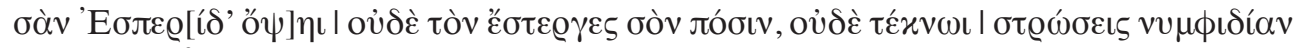

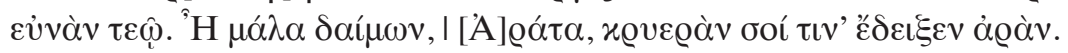

“ Arata fille de Kallikratès, d'Euhespérides. Vieillard, maître de la barque aux bons bordages, qui dans ta navigation éternellement franchis le fleuve sous la nuit ombreuse, as-tu jamais vu une vertu surpassant celle d'Arata, s'il est vrai que tu l'as débarquée sur la rive sinistre?

Jamais plus tu ne verras ta patrie aux enfants délicats, l'Hespéride, ni l'époux que tu chérissais, jamais tu ne prépareras pour ton enfant la couche nuptiale. Ah vraiment, Arata, glaçante est la malédiction que la divinité a fixée pour toi ! ».

Le mari qui dédie probablement cette inscription est seulement cité, sans son nom.

Inscription de Stlakkiè (région de Cyrène, au ${ }^{\text {er }}$ s. apr. J.-C.)

SEG 51, 2212

BE 2004, 447

SEG 53, 2057

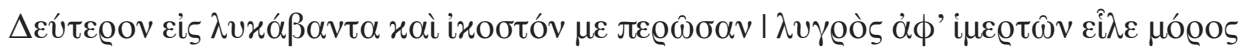

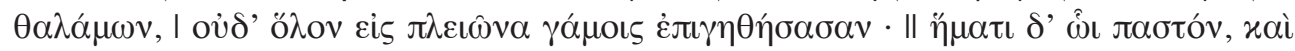

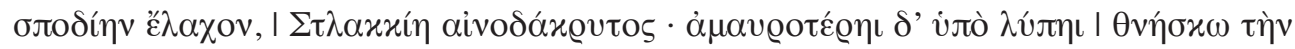

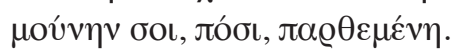

10 Les inscriptions libyennes sont en cours d'étude par Mme Catherine Dobias-Lalou, qui les a traduites et mises aimablement à ma disposition, ce dont je la remercie vivement. 
“ Alors quej'entrais dans ma vingt-deuxième année, un destin funeste m'arracha à mon cher foyer, n'ayant pas même joui de mes noces une année entière : le jour même où m'échut le dais nuptial, m'échut aussi la poussière, lamentable Stlakkiè que je suis ! Et sous le coup d'un chagrin débilitant, je meurs, ne laissant auprès de toi, mon époux, que cette seule fille ».

Inscription de Plauta (région de Cyrène, ${ }^{\mathrm{er}}$-II ${ }^{\mathrm{e}}$ s. apr. J.-C.)

D. M. Robinson, "Inscriptions from the Cyrenaica", American Journal of Archaeology (AJA) 17, 1913, p. 157-200, 161-162

$B E$ 1914, p. 477

$S B$ I, 5873

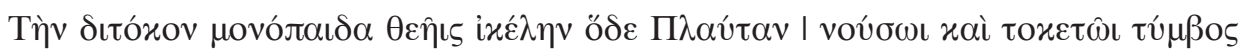

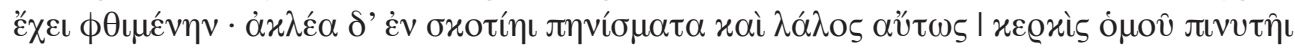

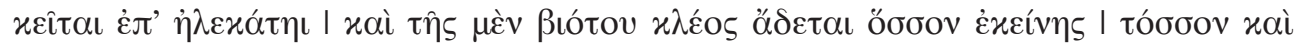

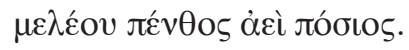

“ Plauta qui deux fois enfanta, mais ne fut qu'une fois mère, Plauta semblable aux déesses, voici le tertre qui l'enferme, décédée du mal d'enfantement ; sans éclat, dans l'obscurité, les tissus qu'elle trama reposent, de même que son fuseau bavard, auprès de sa sage quenouille, et l'on chante autant la renommée de sa vie que la douleur éternelle de son malheureux époux ».

Il est nécessaire de remarquer que la formule initiale " qui deux fois enfanta, mais qui ne fut qu'une fois mère ", plutôt ambiguë, peut se comprendre de deux manières : soit Plauta, déjà mère d'un enfant, est morte lors de son second accouchement ; soit elle est morte lors de son premier accouchement, en mettant au monde des jumeaux.

Comme annoncé plus haut, j’ai cherché dans les inscriptions une forme d'expression directe et spontanée. Si le langage du couple dans les inscriptions funéraires reste sans doute assez conventionnel et presque monotone, il ne semble pas pour autant manquer de sincérité. Et si les phrases et les mots sont formulaires et figés, les sentiments de la douleur, de la perte, du regret de ne plus pouvoir partager les joies et les devoirs de la vie, et surtout les enfants, sont vrais et font partie intégrante du climat familial.

Par ailleurs, remarquons que pour exprimer des sentiments comme l'amour, la douleur, la tristesse, le regret, on utilise encore aujourd'hui des mots ou des phrases assez semblables et qui nous paraissent toujours banals et inadéquats. Mais si les mots sont banals, cela ne veut pas dire que les sentiments ne sont pas sincères ou n'existent pas. 
Cependant, on peut avoir parfois le soupçon que, derrière des mots tellement tendres qu'ils en apparaissent presque grandiloquents, d'autres intentions, moins honnêtes, puissent se cacher.

Un poème voulu pour sa femme morte, Annia Régilla, par Hérode Atticus, nous fait sortir de l'univers des couples anonymes, pour entrer dans la haute société grecque et romaine du $\mathrm{II}^{\mathrm{e}} \mathrm{s}$. apr. J.-C. Hérode Atticus était un riche Athénien, né dans le dème de Marathon. Connu comme orateur et comme mécène, pour avoir fait construire à Athènes et ailleurs en Grèce des édifices grandioses, sous Hadrien et les Antonins, arrivé à Rome sous Antonin le Pieux, il fut adopté par ce dernier et devint le précepteur de ses fils Marc Aurèle et Lucius Verus ; consul en 143, il épousa la riche et noble Annia Régilla. Il a laissé pour sa femme morte brusquement un nombre important d'inscriptions, où il l'honore et la regrette, en Grèce (Athènes, Corinthe et Delphes, où il obtient des décrets de la cité pour l'honorer publiquement) et à Rome.

\section{Poème de Marcellus (voulu par Hérode Atticus) pour Annia Régilla" (deuxième moitié II ${ }^{\mathrm{e}}$ s. apr. J.-C.)}

Froehner 8

IG XIV, 1389

“Venez ici, vers ce temple, ô femmes du Tibre, en emmenant les objets sacrés qui servent pour le sacrifice, autour de la statue de Régilla.

Elle descend des très riches enfants d'Enée, sang illustre d'Anchise et d'Aphrodite du Mont Ida. Elle s'est mariée (pour se rendre) à Marathon, et les déesses célestes, la nouvelle et l'ancienne Déô, l'honorent, et une statue sacrée de (cette) femme à la belle taille est offerte et elle habite parmi les hérö̈nes dans les îles des Bienheureux, là où Cronos règne.

En effet, elle a reçu cette récompense pour ses bons sentiments. Ainsi Zeus eut pitié de l'époux qui se lamentait, couché dans la triste vieillesse sur un lit solitaire, parce que les Fileuses (les Parques), rapaces et noires, avaient enlevé du magnifique palais la moitié de ses enfants ; deux enfants avaient encore été laissés très jeunes, qui ne connaissent pas les malheurs, qui ignorent encore totalement quelle (bonne) mère l'impitoyable destin leur a ravi, avant qu'elle eût touché aux fuseaux de la vieillesse.

A lui (Hérode) qui pleurait sans cesse sa fidèle (épouse), Zeus et l'empereur, qui ressemble au père Zeus pour sa nature et sa sagesse (ont donné cette consolation):

1 Je ne fournis pas le texte grec qui est très long et ne présente aucun mot particulier nécessitant une analyse approfondie. Traduction d'après Froehner, n. 8 (texte grec p. 11-13, traduction p. 15-17). 
Zeus a envoyé la jeune femme à l'Océan, enlevée par les souffles élyséens de Zéphyr; et l'empereur semble avoir accordé à l'enfant les sandales brodées d'étoiles autour des chevilles, que, dit-on, Hermès aussi portait lorsqu'il déroba Enée, une nuit sombre de la guerre des Achéens; autour de ses pieds, il (Hermès) avait un anneau sauveur brillant comme le croissant de lune; les fils d'Enée le cousaient autrefois aux sandales et pour qu'il servît d'ornement honorifique pour les descendants de noble origine.

Cette chaussure, ancienne marque d'honneur des Etrusques, ne l'outragera pas, bien qu'il (Hérode) soit descendant de Cécrops, car il est né d'Hersé et d'Hermès, si c'est vrai que Céryx est l'aïeul d'Hérode le Théséide.

Pour cela il (Hérode) eut les hautes dignités (de sénateur) et le consulat et, certes, (le droit) de se réunir dans l'assemblée souveraine, là où il y a les premiers sièges ; la Grèce ne possède personne plus royale d'Hérode, pour sa famille ou pour son éloquence ; et, en effet, on l'appelle la « langue d'Athènes ».

Et elle aussi, la femme aux belles chevilles, descendant d'Enée et Ganymède, était de la famille de Dardanos et de Trôs, fils d'Erichthonios.

Et toi, si tu le veux bien, offre des sacrifices et immole les victimes (mais il n'est pas nécessaire pour celui qui ne veut pas, d'immoler des victimes) ; mais il semble bien pour les hommes pieux de s'occuper des héros. Et, en effet, elle n'est plus mortelle, mais elle n'est pas encore devenue une déesse ; et elle n'a donc ni temple sacré, ni tombeau, ni les hommages dus aux mortels, ni les honneurs dus aux dieux. Elle a un monument semblable à un temple, chez le peuple d'Athènes, mais son âme sert le sceptre de Rhadamanthe.

Cette statue a été agréée par Faustine, dans le pays de Triopas, là où jadis elle possédait des vastes campagnes, une rangée de vignes et des champs d'oliviers.

L'impératrice, déesse (protectrice) des femmes, ne dédaignera pas d'être la prêtresse de son culte, ni sa nymphe de compagnie.

En effet, ni la déesse qui aime les flèches (Artémis-Diana), assise sur son beau trône, n’a dédaigné Iphigénie, ni Athéna au regard terrible (n’a dédaigné) Hersé, ni la mère bienveillante du puissant César, protectrice des anciennes héroïnes, ne la négligera, elle qui entrera dans le chœur des anciennes demi-déesses, elle qui a eu en sort de diriger l'arrangement des danses élyséennes, avec Alcmène et la fille bienheureuse de Cadmos ».

Il est permis de se demander si dans ce cas, ce poème et les autres inscriptions ne sont pas liés à la nécessité pour Hérode Atticus de dissiper les soupçons qui avaient pesé sur lui après la mort de sa femme. En effet, elle était enceinte de leur cinquième enfant. Le frère de Régilla, Annius Appius Atilius Bradua, avait alors accusé son mari souvent enclin à la colère d'avoir provoqué sa mort, en lui donnant des coups de pieds lors d'une violente dispute. Cité en justice et convoqué à Rome, Hérode dut affronter un procès, qui se termina par un non-lieu pour insuffisance de preuves (mais sa richesse et l'amitié que l'empereur lui vouait 
avaient dû compter alors). Cependant, le nombre de textes qu'il fait graver pour sa femme, et le fait qu'il ait eu recours jusqu'à des institutions publiques pour les faire connaître, font penser qu'il ne devait pas avoir la conscience complètement tranquille, ou qu'il eut à cœur de laver sa réputation.

Si ce document montre que les affaires privées peuvent être étalées publiquement, pour des raisons politiques, je voudrais terminer cette présentation des inscriptions concernant des couples en évoquant trois brefs documents de Delphes : ce sont des dédicaces de statues dans le grand sanctuaire panhellénique, approuvées ou peut-être même sollicitées par le koinon des Amphictions, et dans lesquelles vie publique et vie privée se mêlent et se confondent.

Deux cas peuvent être repérés dans ces inscriptions. Dans le premier texte, le koinon fait la dédicace d'une statue à Caninia Modestina, qui appartient à l'importante famille athénienne des Gellii. Il décide de l'honorer pour ses qualités conjugales, probablement sollicité par son mari, Lucius Gellius Thésée, qui devait être un membre du Conseil amphictionique.

Dans les deux autres inscriptions, des monuments privés sont élevés, mais sur décret des Amphictions, qui permettent à deux femmes de notables de faire des dédicaces (Valeria Catulla, pour son mari l'helladarque Tiberius Claudius Marcellus ; Flavia Amphicléia, pour son mari le proconsul Titus Flavius Phileinos). Cela peut signifier que ces personnes ont demandé l'autorisation de célébrer ceux qu'ils chérissent, ou alors qu'il s'agit d'une consigne des Amphictions, qui, ne disposant plus de grands moyens, encouragent la participation à la vie de la Ligue en jouant sur les relations familiales et de couple.

Ces personnages, dont les femmes sont présentes et jouent un rôle très actif dans ces textes, devaient certainement être des notables qui siégeaient à l'Amphictionie. Ce sont des personnes de qualité, dont les familles sont honorables ; ils aiment leurs femmes, leurs femmes sont respectées et les aiment : de telles vertus méritent l'éloge public.

\section{Inscriptions amphictioniques}

\section{Inscription des Amphictions pour Caninia Modestina}

$\left(2^{\mathrm{e}} \text { moitié du } \mathrm{II}^{\mathrm{e}} \mathrm{s} \text {. apr. J.-C., ou plus tard }\right)^{12}$

CID IV, 160

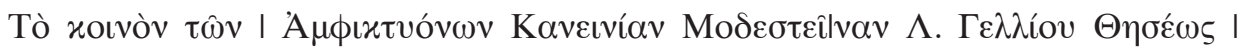

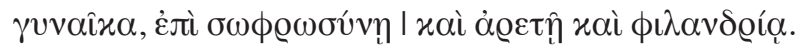

12 Les traductions sont celles du CID IV. 
“ Le koinon des Amphictions (a érigé la statue de) Caninia Modestina, épouse de L. Gellius Thésée, pour sa sagesse, sa vertu et son attachement envers son mari ».

Inscription de Valeria Catulla pour son mari Tiberius Claudius Marcellus $\left(2^{\mathrm{e}}\right.$ moitié II ${ }^{\mathrm{e}} \mathrm{s}$.)

CID IV, 161

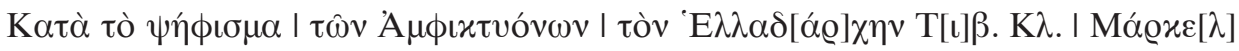

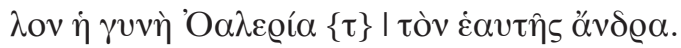

"Conformément au décret des Amphictions, (statue de) l'helladarque Tib. Cl. Marcellus, (érigée par) son épouse Valeria Catulla, (en l'honneur de) son mari ».

Inscription de Flavia Amphicleia pour son mari Titus Flavius Phileinos $\left(1^{\mathrm{er}}\right.$ tiers du $\mathrm{III}^{\mathrm{e}} \mathrm{s}$.)

CID IV, 166

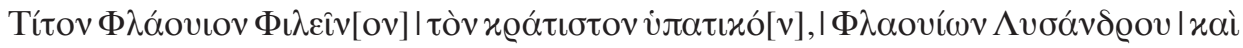

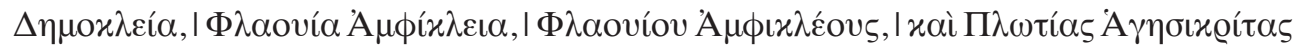

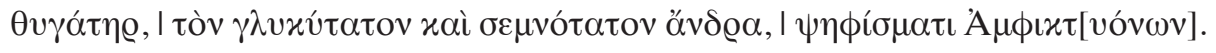

“(Statue de) Titus Flavius Phileinos, le distingué proconsul, fils de Flavius Lysandros et de Flavia Démocléia, (érigée par) Flavia Amphicleia, fille de Flavius Amphiclès et de Plotia Hegèsicritia, (en l'honneur de) son très cher et vénérable époux, sur décret des Amphictions ».

Les vertus honorées sont les mêmes que celles qu'on rencontre dans les inscriptions funéraires d'époque classique ; mais même si ce phénomène était déjà apparu dans les siècles hellénistiques, à l'époque impériale la vie privée fait désormais fréquemment irruption dans la vie publique, avec un langage formulaire et stéréotypé. Soulignons qu'il s'agit d'un aspect qui n'est pas absent de la société d'aujourd'hui. Comme la douleur qui inspire les inscriptions d'éloge et de regret, les intentions politiques, depuis toujours, se manifestent à travers l'apparence que le couple veut donner de ses relations, le statut d'époux ou d'épouse étant important et conférant bonne renommée aux personnages politiques.

Alors je ne peux m'empêcher de penser qu'à bien des égards, l'époque impériale ressemble à celle que nous vivons. Concluons donc sur une note légère, en suggérant que ces femmes d'Amphictions, qui étaient aussi des gens " bien », car ils avaient des femmes " bien ", font songer aux épouses des Présidents ou autres hommes politiques d'aujourd'hui qui, apparaissant souvent dans lapresse, souhaitent ainsi contribuer à donner une image positive de leurs célèbres maris. 
En définitive, l'image des couples grecs, telle que la reflètent les quelques textes inscrits que j'ai cités, ne semble pas différer beaucoup de celle des couples actuels. En tout cas, elle se démarque nettement du jugement négatif que continue de diffuser sur ce point une tradition encore forte dans nos études.

\section{Michela Costanzi}

Maître de conférences en histoire et archéologie grecques à l'UPJV 4, Villa du Parc - 75019 Paris michela.costanzi@u-picardie.fr 